\title{
User-Defined Nonmonotonicity in Unification-Based Formalisms
}

\author{
Lena Strömbäck \\ Department of Computer and Information Science \\ Linköping University \\ S-58185 Linköping, Sweden \\ lestr@ida.liu.se
}

\begin{abstract}
A common feature of recent unificationbased grammar formalisms is that they give the user the ability to define his own structures. However, this possibility is mostly limited and does not include nonmonotonic operations. In this paper we show how nonmonotonic operations can also be user-defined by applying default logic (Reiter, 1980) and generalizing previous results on nonmonotonic sorts Young and Rounds, 1993).
\end{abstract}

\section{Background}

Most of the more recent unification-based formalisms, such as TFS (Emele and Zajac, 1990), UD (Johnson and Rosner, 1989), CUF (Dörre and Eisele, 1991), and FLUF (Strömbäck, 1994), provide some possibility for the user to define constructions of his own. This possibility can be more or less powerful in different formalisms. There are, however, several constructions proposed as desirable extensions to unification grammars that cannot be defined in a general and well-defined way in these formalisms. One such class of constructions is those that have some degree of nonmonotonic behaviour. Examples of such constructions are any-values, default-values, and some constructions (e.g. constraining equations, completeness and coherence) used in LFG Kaplan and Bresnan, 1983).

This paper describes a method that permits the user to define such nonmonotonic constructions. This is done through generalizing the work on nonmonotonic sorts (Young and Rounds, 1993). This generalization results in a default logic similar to (Reiter, 1980), but where subsumption and unification are used instead of logical truth and consistency.

There are three main advantages to Young and Rounds' work compared with other approaches to default unification (Bouma, 1990; Bouma, 1992; Russel et al., 1992 which justify choosing it as a starting point for this work. The first is the separation of definite and default information, where Young and Rounds are more distinct than the other. The second is that the nonmonotonic unification operation used is order independent. This is achieved by separating the unification operation from computing the nonmonotonic extension, which Young and Rounds call explanation. This suggests that all the user needs to define when generalizing the approach is how a sort is explained. Finally, there is a close relationship to Reiter's (1980) default logic.

This paper starts by providing the minimal properties required of a unification-based formalism when extending with nonmonotonic definitions. I then describe the approach of user-defined nonmonotonicity and illustrate how some commonly used nonmonotonic constructions can be defined within it. Finally I conclude with a discussion of the relation to Reiter's default logic and computational properties of the approach.

\section{Preliminaries}

There are two main properties that will be assumed of a unification-based formalism in order to extend it with the possibility of defining nonmonotonic constructions. The first, and most important, is that we require a subsumption order on the set $\mathcal{S}$ of objects denoted by the formalism. Secondly it should be possible to define inheritance hierarchies on the linguistic knowledge described by the formalism.

One very plausible subsumption order that can be used is the ordinary subsumption lattice of feature structures. It is, however, possible to use some other kind of subsumption order if that is more suitable for the domain to be modelled by the formalism. Examples of other subsumption orders that might be useful are typed feature structures, feature structures extended with disjunction, or simply an order based 
on sets and set inclusion.

In this paper the notation $a \sqsubseteq b$ is used whenever $a$ subsumes $b$ (i.e. whenever $a$ "is more specific than" or "contains more information than" $b$ ). Consequently, $a \sqsubset b$ is used whenever $a \sqsubseteq b$ but $a \neq b$.

The subsumption order is assumed to be a semilattice and permits computing a unifier, denoted $a \sqcap b$, corresponding to the greatest lower bound, for every pair of elements within it. The element corresponding to the bottom of the order relation is denoted fail and represents inconsistent information or unification failure.

The second constraint placed on the formalism, the possibility of defining an inheritance hierarchy, is not essential for the definition of nonmonotonic operations. It is, however, very useful when defining nonmonotonic constructions. The following notation will be used for describing an inheritance hierarchy.

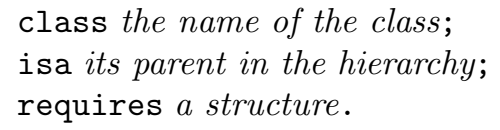

Thus, each member in the inheritance hierarchy is called a class, which is defined by giving it a name and a parent in the hierarchy. It is also possible to define some constraints, called requirements, which must hold for a class. These requirements can be both structures in the subsumption order and nonmonotonic rules. The constraints on classes are inherited through the hierarchy. Every object in a class is assumed to contain at least the information given by the constraints specified for it and all its ancestors. For simplicity multiple inheritance between classes will not be allowed. This means that two classes where none of them is a subclass of the other, will always be considered inconsistent and thus yield a failure when unified.

\section{User-Defined Nonmonotonicity}

I will now describe how the work by Young and Rounds can be generalized to allow the user to define nonmonotonic constructions. The main idea in their approach is that each node in a feature structure consists of a nonmonotonic sort. Such sorts can contain two different kinds of information, the ordinary monotonic information and a set of defaults. If we assume that $\beta$ is defined as a default in Young and Rounds' work then it is interpreted according to the rule: if it is consistent to believe $\beta$ then believe $\beta$. In Reiter's default logic this is expressed with the following normal default rule.

$$
\frac{: \beta}{\beta}
$$

In this paper I want to allow the user to use other forms of nonmonotonic inferences and not only the normal default rule given above. Therefore, I will consider the general form of default rules. An intuitive reading of a general default rule is, if $\alpha$ is believed and it is consistent to believe $\beta$ then believe $\gamma$. In default logic this is usually expressed as

$$
\frac{\alpha: \beta}{\gamma}
$$

The next question is how such defined nonmonotonic rules are going to be interpreted in a unification framework. In (Reiter, 1980), a rule like the one above could be applied whenever $\alpha$ is true and $\beta$ is consistent with the information we already have. If we assume that $\mathcal{V}$ represents the information already given this means that the default rule can be applied whenever $\mathcal{V} \sqsubseteq \alpha$ and $\mathcal{V} \sqcap \beta$ does not yield unification failure. When the rule is applied the new information obtained would be $\mathcal{V} \sqcap \gamma$.

In the approach described in this paper, the user is allowed to define the actual nonmonotonic rule that should be used for a particular operation by using the following syntax.

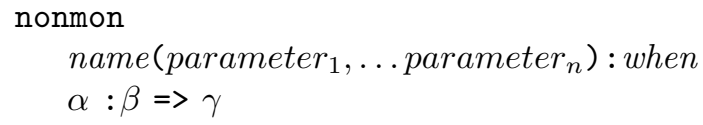

In the syntax given above name assigns a name to the defined rule, and thus allows the user to use nonmonotonic information when defining linguistic knowledge. The parameters in the rule definition are variables, which can be used within the actual default rule at the end of the description. The user is assumed to assign the nonmonotonic information contained in this rule to his linguistic knowledge by using an expression of the form name $\left(\right.$ parameter $_{1}, \ldots$ parameter $\left._{n}\right)$.

The when slot in the rule allows the user to decide when the rule is going to be applied, or in Young and Rounds' terminology, explained. I will make use of two values for the when-slot, immediate and posterior. Immediate means that the nonmonotonic rule is going to be applied each time a full unification task has been solved or whenever all information about an object in the defined inheritance hierarchy has been retrieved. Posterior explanation means that the explanation of the rule is postponed until reaching the result of some external process, for example, a parser or generator. There is however no hinder in excluding the use of other values here. One could, for example, imagine cases where one would want different nonmonotonic rules to be explained after 
a completed parse, a generation, or after resolving discourse referents.

Note that although the when slot in the definition of a nonmonotonic rule allows the user to define when his rule is going to be applied we will still have an order independent nonmonotonic unification operator. This is the case because we follow Young and Rounds' approach and separate the unification operation from the explanation of a nonmonotonic rule. Therefore, what affects the final result of a computation is when one chooses to explain default rules and not the order of the unification operations occurring between such explanations.

\section{Formal Definitions}

In this section I provide give the formal definitions for nonmonotonic sorts and how nonmonotonic sorts are unified and explained. The definitions are generalizations of the definitions in Young and Rounds (1993). The notation $a \sim b$ is used to denote the fact that $a \sqcap b$ does not yield unification failure.

A nonmonotonic sort is a structure containing both information from the basic subsumption order and information about default rules to be explained at a later point in the computation.

Definition 1 A nonmonotonic sort is a pair $\langle s, \Delta\rangle$ where $s \in \mathcal{S}$ and $\Delta$ is a set of nonmonotonic rules of the form $\langle w, \alpha: \beta \Rightarrow \gamma\rangle$ where $w$ is an atom and $\alpha, \beta$ and $\gamma \in \mathcal{S}$. It is assumed that for each nonmonotonic rule $\gamma \sqsubseteq \beta, \alpha \sim s, \beta \sim s$, and $\gamma \sqcap s \sqsubset s$.

As seen by the definition a nonmonotonic sort is considered to be a pair of monotonic information from the subsumption order and nonmonotonic information represented as a set of nonmonotonic rules. The user can assign nonmonotonic information to a nonmonotonic sort by calling a nonmonotonic definition as defined in the previous section. The actual nonmonotonic rule occurring within the sort is a pair consisting of the when slot and the last part of the nonmonotonic definition, with the parameter variables instantiated according to the call made by the user.

The second part of this definition contains some well-foundedness conditions for a nonmonotonic sort. The first condition $(\gamma \sqsubseteq \beta)$ is a restriction similar to the restriction to normal default rules in Reiter's (1980) default logic. This restriction ensures that the application of one default rule will never cause previously applied default rules to be inapplicable. This makes the procedure for application of defaults more efficient and will be further discussed in section 6 .
The next two conditions in the definition, $\alpha \sim s$ and $\beta \sim s$, guarantee that the default rule is or can be applicable to the nonmonotonic sort. The reason for only checking that $\alpha \sim s$ instead of $s \sqsubseteq \alpha$ is that future unifications can restrict the value of $s$ into something more specific than $\alpha$ and thus may make the default rule applicable.

The last condition on a nonmonotonic sort, $\gamma \sqcap s \sqsubset$ $s$, may seem superfluous. The reason for including it is to ensure that applying the default actually restricts the value of the sort. Otherwise the default rule would have no effect and can be removed. Note in particular that the above conditions on a nonmonotonic sort implies that $\gamma$ may be fail.

Given the unification operation of objects within the subsumption order and the definition of nonmonotonic sorts it is possible to define an operation for nonmonotonic unification.

Definition 2 The nonmonotonic unification $\left(\Pi_{N}\right)$ of two nonmonotonic sorts $\left\langle s_{1}, \Delta_{1}\right\rangle$ and $\left\langle s_{2}, \Delta_{2}\right\rangle$ is the sort $\langle s, \Delta\rangle$ where

$$
\begin{aligned}
& \text { - } s=s_{1} \sqcap s_{2} \text { and } \\
& \text { - } \Delta=\left\{d \mid d=\langle w, \alpha: \beta \Rightarrow \gamma\rangle, d \in \Delta_{1} \cup\right. \\
& \left.\Delta_{2}, \alpha \sim s, \beta \sim s, \text { and } \gamma \sqcap s \sqsubset s\right\}
\end{aligned}
$$

The nonmonotonic unification is computed by computing the unification of the monotonic parts of the two sorts and then taking the union of their nonmonotonic parts. The extra conditions used when forming the union of the nonmonotonic parts of the sorts are the same as in the definition of a nonmonotonic sort and their purpose is to remove nonmonotonic rules that are no longer applicable, or would have no effect when applied to the sort resulting from the unification.

It is important to note that this generalization of the original definition of nonmonotonic unification from Young and Rounds (1993) preserves the property of order independence for default unification.

When using nonmonotonic sorts containing nonmonotonic rules, we also need to know how to merge the monotonic and nonmonotonic information within the sort. I will use the terminology $w$ application for applying one nonmonotonic rule to the sort and w-explanation when applying all possible rules.

Definition 3 The nonmonotonic rule $\langle w, \alpha: \beta \Rightarrow \gamma\rangle$ is $w$-applicable to $s \in \mathcal{S}$ if:

$$
\begin{aligned}
& \text { - } s \sqsubseteq \alpha \\
& \text { - } s \sim \beta \text { or } s=\text { fail } \\
& \text { - } s \sqcap \gamma \sqsubset s \text { or } s=\text { fail }
\end{aligned}
$$


The result of the $w$-application is $\gamma \sqcap s$

Note that the $w$ in $w$-application should be considered as a variable. This means that only nonmonotonic rules whose first component is $w$ are considered and that it is possible to choose which nonmonotonic rules should be applied in a particular point at some computation.

In addition note that the restriction that $\gamma \sqsubseteq \beta$ in all nonmonotonic rules and the special cases for $s=$ fail ensures that the application of one nonmonotonic rule never destroys the applicability of a previously applied rule. This reduces the amount of work required when computing a w-explanation. Based on these observations, a sufficient condition for w-explanation is defined as follows.

Definition $4 t$ is a w-explanation of a nonmonotonic sort $\langle s, \Delta\rangle$ if it can be computed in the following way:

1. If $s=$ fail or no $d \in \Delta$ is $w$-applicable then $t=s$ else

2. Choose a $d=\langle w, \alpha: \beta \Rightarrow \gamma\rangle \in \Delta$ such that $d$ is $w$-applicable to $s$.

3. Let $s=s \sqcap \gamma$ and go to 1 .

As shown by the definition, a w-explanation is computed by choosing one w-applicable default rule at a time and then applying it. Since the definition of w-applicability and the condition that $\gamma \sqsubseteq \beta$ in all nonmonotonic rules ensures that whenever a nonmonotonic rule is applied it can never be inapplicable, there is no need to check if the preconditions of earlier applied nonmonotonic rules still hold.

Note also that the choice of which nonmonotonic rule to apply in each step of a w-explanation is nondeterministic. Consequently, it is possible to have conflicting defaults and multiple w-explanations for a nonmonotonic sort.

Note also that the result of a w-explanation is allowed to be fail. Another option would be to interpret fail as if the application of the nonmonotonic rule should not be allowed. However, as seen in the next section, for many uses of nonmonotonic extensions within unification-based formalisms, the aim is to derive failure if the resulting structure does not fulfill some particular conditions. This makes it important to allow fail to be the result of applying a nonmonotonic rule.

\section{$5 \quad$ Examples}

In this section I will show how some of the most common nonmonotonic extensions to unificationbased grammar can be expressed by defining rules as above. I will start with defining default values. This is done by defining a nonmonotonic rule default for the class value, which is assumed to be the most general class in a defined hierarchy. The rule defined here is treated as the one in (Young and Rounds, 1993 ).

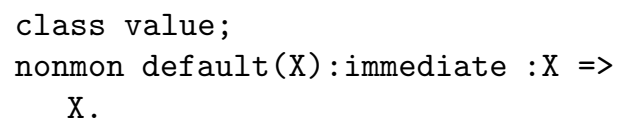

This default rule can be used when defining verbs. The rule is used for stating that verbs are active by default. I also define the two Swedish verbs skickade (sent) and skickades (was sent) to illustrate how this rule works.

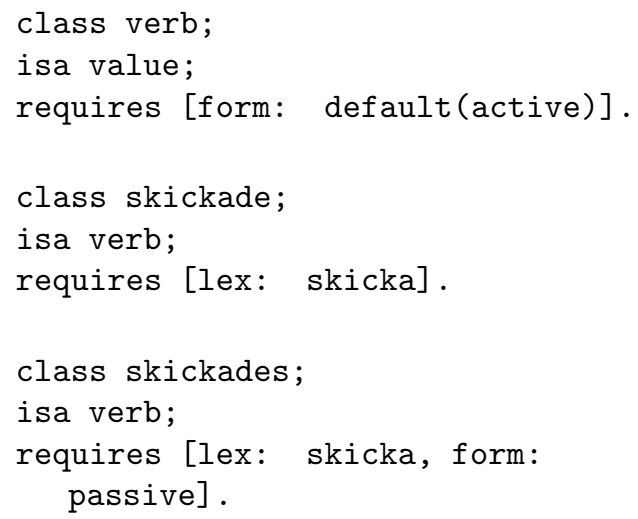

While retrieving the information for these two verbs we will obtain the following two feature structures containing nonmonotonic sorts:

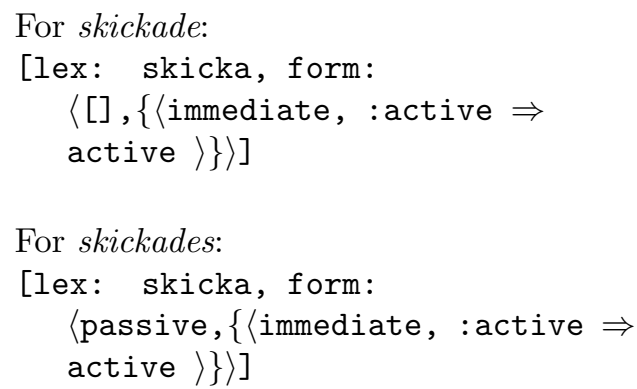

Since I have used immediate for the when-slot in the definition of the default rule, this nonmonotonic rule will be applied immediately after retrieving all information about a verb in the hierarchy. For the two structures above, the default rule can be applied for skickade, since active is consistent with [], but not for skickades, since active and passive are inconsistent. The result after applying immediateexplanation to the two structures above is shown below. 
For skickade:

[lex: skicka, form: active]

For skickades:

[lex: skicka, form: passive]

Another nonmonotonic operation that has been used in LFG (Kaplan and Bresnan, 1983) is the value constraint $(=c)$ used to check whether a substructure has a particular value after a completed parse. The definition of value constraints as a nonmonotonic rule makes use of negation, interpreted as negation as failure.

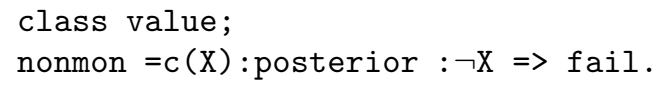

One use of value constraints in LFG is to assert a condition that some grammar rules can only be used for passive sentences. I will here assume that a representation for verbs where passive verbs have the value passive for the attribute form, but where other verbs have no value for this attribute. In the syntax used in this paper the constraint that a particular grammar rule can only be used for passive verbs would be expressed as below:

\section{[form: $=c($ passive $)]$}

This would result in the nonmonotonic sort:

$$
\begin{aligned}
& \text { [form: }\langle[],\{\langle\text { posterior, }: \neg \text { passive } \\
& \quad \Rightarrow \text { fail }\rangle\}\rangle]
\end{aligned}
$$

As seen by the definition of $=c$, the explanation for this nonmonotonic sort is postponed and is assumed to be computed after finding a parse for some sentence. This implies that the only case where this rule would not apply, and thus not give fail as a result, is when the value of form actually is passive. For all other values of form, we would have something that is consistent with $\neg$ passive and thus the nonmonotonic rule will derive failure when applied.

The next nonmonotonic structure I want to discuss is any-values. The inheritance hierarchy is used to be able to define any-values in a simple way.

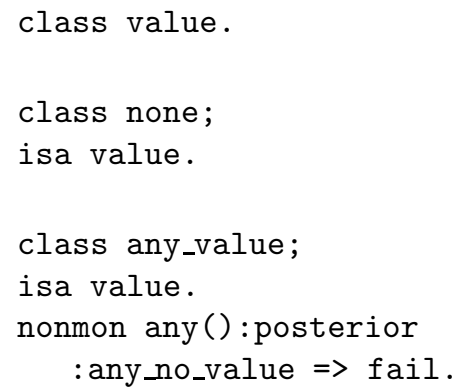

class any_no_value;

isa any_value.

In this small hierarchy it is assumed that all possible values of a structure is a subtype of value. We then divide this into none, which represents that a structure cannot have any value and any_value which contains all actual values. The class any_value is then further divided into a class called any_no_value, which only contains this single value, and the actual values of a structure. The class any_no_value should not be used when defining linguistic knowledge. However, when applying the default rule a value that has not been instantiated is compatible with this any_no_value. Therefore the default rule can make the conclusion that the structure is inconsistent, which is what we desire. Note that, as soon as a value has been further instantiated into a 'real' value, it will no longer be consistent with any_no_value, and the nonmonotonic rule cannot apply. Two examples will further illustrate this.

The nonmonotonic sort:

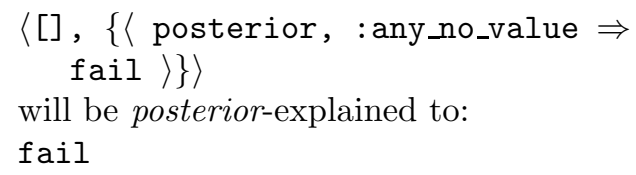

While the sort:

〈[lex: kalle], \{〈 posterior, : any_no_value $\Rightarrow$ fail $\rangle\}\rangle$

will be posterior-explained to:

[lex: kalle]

The last nonmonotonic operations I want to discuss are completeness and coherence as used in LFG. To be able to define these operations I assume the inheritance hierarchy above, without the nonmonotonic definition of any. I will, instead, make use of the two nonmonotonic definitions below.

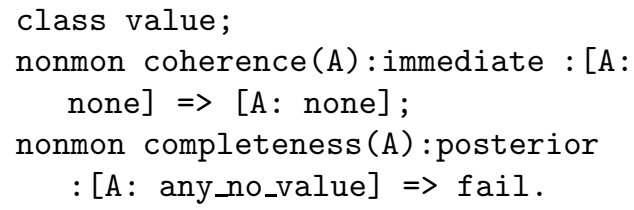

The first of these rules is used to check coherence, and the effect is to add the value none to each attribute that has been defined to be relevant for coherence check, but has not been assigned a value in the lexicon. The second rule is used for checking completeness and it works similarly to the anydefinition above.

Finally, I will show how a fragment of a lexicon can be defined according to these rules. Note that in the 
definition of the transitive verb, the value any_value is given to the appropriate attributes. This means that they are inconsistent with none, and thus, the coherence rule cannot be applied.

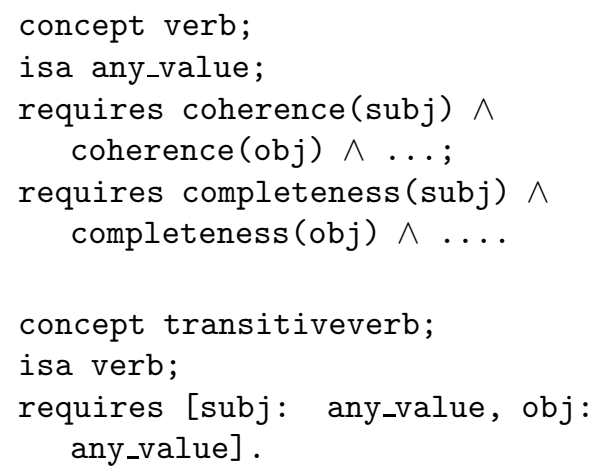

\section{Relation to Default Logic}

In this section I will discuss the relation of this work to Reiter's (1980) default logic. There will also be some discussion on the computational properties and limitations of the given approach.

Compared with Reiter's default logic, our notion of nonmonotonic sorts corresponds to default theories. Unification of nonmonotonic sorts would then correspond to merging two default theories into one single theory and our notion of explaining a nonmonotonic sort corresponds to computing the extension of a default theory in default logic.

In default logic there is often a restriction to normal-default theories since non-normal default theories are not even semi-decidable. The restriction in our nonmonotonic rules that $\gamma \sqsubseteq \beta$ is similar to the restriction into normal default rules and captures the important property, that the application of one nonmonotonic rule should not affect the applicability of previously applied rules. The decidability of the nonmonotonic rules defined here is, however, highly dependant on the given subsumption order. In particular it is dependent on having a decidable unification operation and subsumption check.

As mentioned previously there is with nonmonotonic sorts, as well as normal default logic, a possibility of conflicting defaults and thus multiple nonmonotonic extensions for a structure. One difference is that nonmonotonic sorts allow that the application of a nonmonotonic rule leads to fail, i.e. an inconsistent structure, while default logic does not allow this outcome. However, since fail is allowed as a valid explanation for a nonmonotonic sort, there is, as for normal default logic, always at least one explanation for a sort.

The two following examples will illustrate the difference between nonmonotonic rules giving multiple extensions and nonmonotonic rules giving a single explanation fail.

Example a

$$
\frac{:[a: 1]}{[a: 1 b: 1]} \quad \frac{:[c: 2]}{[b: 2 c: 2]}
$$

Example b

$$
\frac{:[a: 1]}{[a: 1 b: 1]} \quad \frac{:[b: 2]}{[a: 2 b: 2]}
$$

In example $a$ the application of one rule, does not make the other inapplicable. Thus the only explanation for a structure is achieved by applying both these two rules and results in fail. In example $b$, however, the application of one of the rules would block the application of the other. Thus, in this case there are two explanations for the structure dependant on which of the rules that has been applied first. Note that even though there is an order dependency on the application order of nonmonotonic rules this does not affect the order independency on nonmonotonic unification between application of nonmonotonic rules.

Allowing multiple extensions gives a higher computational complexity than allowing only theories with one extension. Since it is the user who defines the actual nonmonotonic theory multiple extensions must be allowed and it must be considered a task for the user to define his theory in the way he prefers.

\section{Improvements of the Approach}

I will start with two observations regarding the definitions given in section 3 . First, it is possible to generalize these definitions to allow the first component of a nonmonotonic sort to contain substructures that are also nonmonotonic sorts. With the generalized versions of the definitions explanations that simultaneously explain all substructures of a nonmonotonic sort will be considered. Note that the explanation of default rules at one substructure might affect the explanation of rules at other substructures. Therefore the order on which nonmonotonic rules at different substructures are applied is important and all possible application orders must be considered.

Considering unification of nonmonotonic sorts it is not necessary to simplify the nonmonotonic part of the resulting sort. $\Delta=\Delta_{1} \cup \Delta_{2}$ can be defined as an alternative to the given definition. This alternate definition is useful for applications where the simplification of nonmonotonic sorts by each unification is expected to be more expensive than the extra work 
needed to explain a sort whose nonmonotonic part is not simplified.

As stated previously, nonmonotonic sorts allow multiple explanations of a nonmonotonic sort. If desired, it would be fairly easy to add priorities to the nonmonotonic rules, and thus induce a preference order on explanations.

One further example will illustrate that it is also possible to define negation as failure with nonmonotonic rules. An intuitive interpretation of the defined rule below is that if $X$ is believed $(\mathcal{V} \sqsubseteq X)$, failure should be derived.

nonmon not $(X)$ : immediate $X=>$ fail;

However, if this definition is to be really useful we must also allow one definition of a nonmonotonic rule to make use of other nonmonotonic rules. In our original definition we said that the nonmonotonic rule above should be applied if $\mathcal{V} \sim \neg X$. This can be generalized to the case where $\neg X$ is a nonmonotonic rule if we extend the definition of $\sim$ to also mean that the application (or explanation) of the not rule at this node does not yield failure. However, this generalization is outside default logic. Therefore, its computational properties are unclear and needs more investigation.

\section{Conclusion}

In this paper I have proposed a method allowing the user to define nonmonotonic operations in a unification-based grammar formalism. This was done by generalizing the work on nonmonotonic sorts (Young and Rounds, 1993) to allow not only normal defaults rules but general default rules that are defined by the user. The method has a very close relation to Reiter (1980). We also noted that the method can be applied to all domains of structures where we have a defined subsumption order and unification operation.

The generality of the approach was demonstrated by defining some of the most commonly used nonmonotonic operations. We also gave formal definitions for the approach and provided a discussion on its computational properties.

\section{Acknowledgments}

This work has been supported by the Swedish Research Council for Engineering Sciences (TFR). I would also like to thank Lars Ahrenberg and Patrick Doherty for comments on this work and Mark A. Young for providing me with much-needed information about his and Bill Rounds' work.

\section{References}

[Bouma1990] Gosse Bouma. 1990. Defaults in Unification Grammar. in Proceedings of the 1990 Conference of the Association for Computational Linguistics, pages 165-172.

[Bouma1992] Gosse Bouma. 1992. Feature Structures and Nonmonotonicity. Computational Linguistics 18(2):183-203.

[Dörre and Eisele1991] Jochen Dörre and Andreas Eisele. 1991. A Comprehensive Unification-Based Grammar Formalism. DYANA Report - Deliverable R3.1B. January 1991.

[Emele and Zajac1990] Martin C. Emele, and Remi Zajac. 1990. Typed Unification Grammars. In Proceedings of the 13th International Conference on Computational Linguistics, Vol. 3, pages 293298, Helsinki, Finland.

[Johnson and Rosner1989] Rod Johnson and Michael Rosner. 1989. A Rich Environment for Experimentation with Unification Grammars. In Proceedings of the 4th Conference of the European Chapter of the Association for Computational Linguistics, pages 182-189, Manchester, England.

[Kaplan and Bresnan1983] R. Kaplan and J.Bresnan. 1983. A Formal System for Grammatical Representation. In: J Bresnan (ed.), The Mental Representation of Grammatical Relations. MIT Press, Cambridge, Massachusetts.

[Reiter1980] Ray Reiter. 1980. A Logic for Default Reasoning. In Artificial Intelligence, 13:81-132.

[Russel et al.1992] Graham Russel, Afzal Ballim, John Carrol and Susan Warwick-Armstrong. 1992. A Practical Approach to Multiple Default Inheritance for Unification-Based Lexicons. Computational Linguistics 18(3):311-337.

[Strömbäck1994] Lena Strömbäck. 1994. Achieving Flexibility in Unification Grammars. In Proceedings of the 15th International Conference on Computational Linguistics, Vol. 3, pages 842-846, Kyoto, Japan.

[Young and Rounds1993] Mark A Young and Bill Rounds. 1993. A Logical Semantics for Nonmonotonic Sorts. In Proceedings of the 1993 Conference of the Association for Computational Linguistics, pages 209-215 